Methods: Patients with a diagnosis of BD who visited our outpatient clinic until December 2019 were included in the study. Clinical data were recorded since diagnosis until the latest follow-up visit, analyzing clinical features, flares and therapeutic strategies adopted.

Results: A total of 95 patients were included in the study with a medium follow-up of $108.54 \pm 169.59$ months. 20 of them (21. 05\%) were treated with biologic agents. Patients treated with biologic therapy compared to those on conventional non-biologic therapies had a higher proportion of musculoskeletal ( $80 \%$ vs $46.67 \%, p=0.008)$, neurological ( $30 \%$ vs $10.67 \%, p=0.031)$, intestinal involvement ( $40 \%$ vs $12 \%, p=0.004)$, and they were treated with a higher dose of glucocorticoids at diagnosis $(16.84 \mathrm{mg} \pm 14.01 \mathrm{vs} 8.89 \mathrm{mg} \pm 11.76, \mathrm{p}=0.012$ ). The most frequent indications for biologic step-up therapy were musculoskeletal involvement $(40 \%)$, eye involvement $(25 \%)$, neurological involvement $(15 \%)$ and intestinal involvement (10\%). Most patients initiated a biologic treatment within the first year of follow-up. TNF-inhibitor (TNFi) were more frequently prescribed (95\%) and one patient was treated with 8 therapeutic cycles of Rituximab ( $500 \mathrm{mg} /$ weekly for 4 infusions to be repeated after at least 6 months) because of recurrent pancytopenia. All patients experienced non-biologic therapy before starting a TNFi. The preferred first-line TNFi was infliximab $(50 \%)$, followed by adalimumab (40\%) and etanercept (5\%). As second line treatment were also prescribed certolizumab (10\%) and golimumab (5\%). 10 patients switched to a second line treatment because of inefficacy of the first biologic agent, mainly because of refractory arthritis, intestinal and mucocutaneous involvement. One patient switched from infliximab to certolizumab during pregnancy with subsequent worsening of arthritis.

$85 \%$ of patients treated with biologic agents reached a clinical remission by the time of the latest follow up visit without any safety or tolerability issues.

Conclusion: A relevant proportion of patients in our BD cohort were treated with biologic therapy, because of severe or refractory manifestations. The most frequent indications were musculoskeletal, neurological or intestinal involvement. Biologic agents were a generally effective and safe therapeutic approach

References:

[1] F. Alibaz-Oner, M. H. Sawalha, H. Direskeneli. Management of Behçet disease, Curr. Opin. Rheumatol, 2018

Table 1. General characteristics and disease involvement at diagnosis

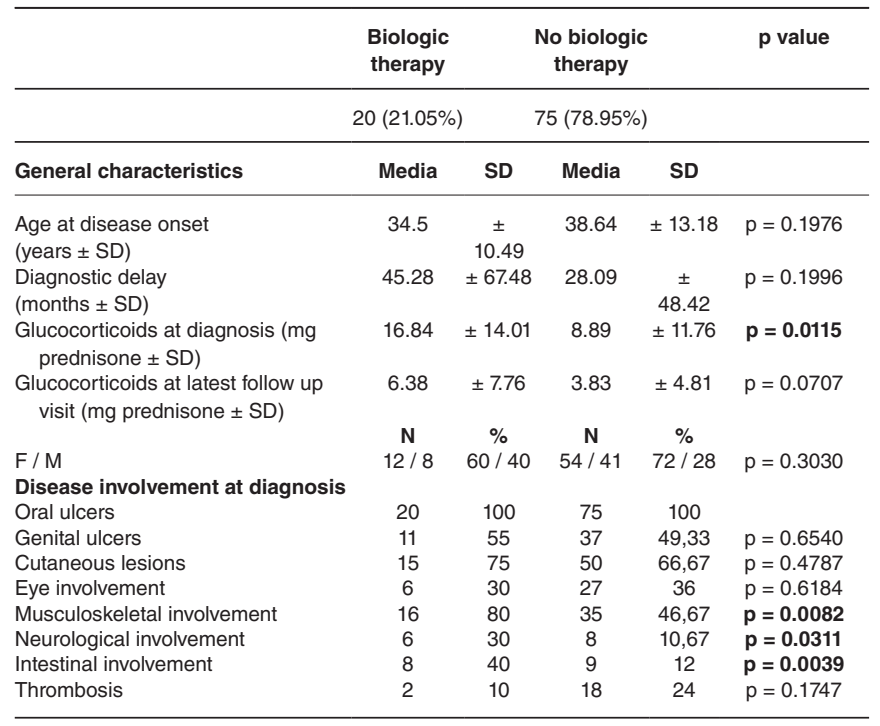

Disclosure of Interests: None declared

DOI: 10.1136/annrheumdis-2020-eular.4582

\section{AB0463 ETIOLOGY OF PALPABLE PURPURA; A SINGLE CENTER EXPERIENCE}

G. Kaynar ${ }^{1}$, O. C. İçaçan ${ }^{1}$, S. Çelik ${ }^{1}$, M. Yalçın Mutlu ${ }^{1}, \underline{\text { C. Bes }}{ }^{1} .{ }^{1}$ University of Health Sciences, Bakırköy Dr. Sadi Konuk Training and Research Hospital, Department of Rheumatology, Istanbul, Turkey

Background: Purpura are lesions that occur after bleeding on the skin, mucous or serosal surfaces. Purpura can be classified into 2 subgroups; which are linked to thrombocytopenia and non-trombocytopenic purpura. While thrombocytopenic purpura often occurs due to a hematological disease; in non-trombocytopenic purpura etiological causes are very variable, and systemic vasculitis has an important place among them.
Objectives: The demographic features of the patients applying with purpura and the underlying causes and diseases were aimed to be revealed.

Methods: 44 consecutive patients ( 22 women, 22 men) who were admitted to the hospital in the last 6 months, due to purpura were evaluated. Average age of patients was $49.6 \pm 19.6$ years. Patients were questioned about a recent infection, drug use, concomitant or underlying diseases. The serological tests and other laboratory tests for etiology were performed and biopsy was taken from the skin lesions which are appropriate.

Results: While 4 (9\%) patients had widespread purpura on the trunk-upperlower extremities, in $22(50 \%)$ patients purpuric lesions were limited only to the lower limb. The period between the onset of the first symptom and the admission to the hospital was longer than 4 weeks in $35(79.5 \%)$ patients, and shorter than 1 week in $9(20.5 \%)$ patients. $24(54 \%)$ patients had an anamnesis of infection 2-3 weeks before purpura, and $20(45 \%)$ patients had an anamnesis of drug use. The most common accompanying symptom was abdominal pain and was present in 15 (34\%) patients. Biopsy was performed from the skin lesion in 37 patients. Histopathological examination of all was compatible with leukocytoclastic vasculitis. In indirect immunofluorescence staining, 17 were found to be IgA positive. $2(4.5 \%)$ patients were diagnosed PR3-ANCA positive granulomatosis with polyangiitis. 1 patient had Hepatitis $B$ virus infection was detected in 1 patient $(2.2 \%)$, HIV infection was detected in 1 patient $(2.2 \%)$ and malignancy was detected in 1 patient $(2.2 \%)$.

Conclusion: In our study, the most common reason was found as IgA vasculitis in patients presenting with palpable purpura. Although vasculitic involvement was limited to the skin in most patients, organ-threatening systemic vasculitis was detected in a few patients. Patients applying with Purpura should be questioned for infection and drug use, should be examined for underlying diseases including systemic vasculitis, and closely monitored for organ involvement.

Disclosure of Interests: None declared

DOI: 10.1136/annrheumdis-2020-eular.5471

\begin{tabular}{|l|l}
\hline AB0464 & THE ROLE OF CYCLOPHOSPHAMIDE \\
CHEMOTHERAPY IN THE TREATMENT OF \\
EOSINOPHILIC GRANULOMATOSIS WITH \\
POLYANGIITIS
\end{tabular}

B. Bitik ${ }^{1}$, M. Aydın ${ }^{2}$, G. Sahin Dalgic ${ }^{3}$, D. Kaskari ${ }^{4}$, A. E. Yucel ${ }^{3} .{ }^{1}$ Baskent University, Ankara, Turkey; ${ }^{2}$ Baskent University, Adana, Turkey; ${ }^{3}$ Baskent University, Ankara, Turkey; ${ }^{4}$ Baskent University, Istanbul, Turkey

Background: Eosinophilic granulomatosis with polyangiitis (EGPA) is a rare type of ANCA associated Vasculitis (AAVs). Cyclophosphamide (CYC) is generally recommended for the induction of remission in life/organ threatening $A A V s$ in combination with glucocorticoids. However, due to its rarity, randomized controlled trials regarding the efficacy of treatment modalities in EGPA are hard to perform. Therefore, the level of evidence for the use of CYC in the treatment of EGPA is lower when compared to other AAVs (1).

Objectives: The aim of this study is to investigate common therapeutic agents used for the treatment of patients with EGPA.

Methods: Medical records of patients who were followed-up with the diagnosis of EGPA between 2007-2020, in rheumatology clinics of Ankara and Adana Hospitals of Başkent University, were analyzed retrospectively. Treatment outcomes were assessed.

Results: Records of 11 patients (six females) were analyzed. The median age was 47 (19-77) years. The median follow-up time of the patients was 24 (9-156) months. Six patients were diagnosed with asthma. The median time between the diagnosis of asthma and EGPA was 4.5 (1-3) years. Five patients had tissue biopsies. Biopsy locations were terminal ileum, lung, myocardium and nerve. The most common forms of involvement were asthma, eosinophilic pneumonia and / or nodule, cardiovascular involvement, mononoritis multiplex, vasculitic skin rash, arthritis and bowel involvement, respectively. P-ANCA was positive in 8 patients. Three patients had myocarditis and cardiomyopathy, and two patients had isolated valve problems. The median BVAS value at the time of diagnosis and the third month of treatment was 17 (6-27) and 4 (2-7), respectively.

Nine patients used oral $1 \mathrm{mg} / \mathrm{kg}$ methylprednisolone (MP) and 500mg CYC every two weeks as an induction therapy. The cumulative median CYC dose was $4.5 \mathrm{~g}(1.5-8)$. Neither of the patients developed CYC related side effects. MP was tapered to $2 \mathrm{mg}$ in five patients, and was quited in two patients. Azathioprine (AZA) was used in remission treatment following CYC therapy. Rituximab (RTX) therapy $1 \mathrm{~g}$ twice, 2 weeks apart was initiated in two patients due to unresponsiveness to CYC. While RTX was effective in one patient, newly developed renal involvement was detected after the third cycle of RTX therapy in other patient. Two patients had pregnancy plan therefore they used AZA plus MP as induction. A patient had mycophenolate mofetil plus MP due to AZA allergy. All patients are currently in remission except one patient.

Conclusion: In seven out of 11 EGPA patients, long-term remission was achieved with CYC treatment. CYC appears to be an effective and inexpensive method of first-line treatment for organ threatening EGPA. 